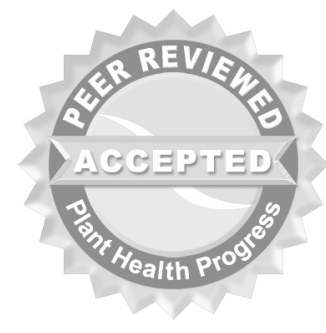

(c) 2008 Plant Management Network.

Accepted for publication 21 December 2007. Published 18 March 2008.

\title{
A Synopsis of Phytophthora with Accurate Scientific Names, Host Range, and Geographic Distribution
}

Erica T. Cline, Research Associate, David F. Farr, Research Scientist, and Amy Y. Rossman, Research Scientist, Systematic Mycology \& Microbiology Laboratory, USDA Agricultural Research Service, 10300 Baltimore Avenue, Beltsville, MD 20705-2350

Corresponding author: Amy Y. Rossman. Amy.Rossman@ars.usda.gov

Cline, E. T., Farr, D. F., and Rossman, A. Y. 2008. A synopsis of Phytophthora with accurate scientific names, host range, and geographic distribution. Online. Plant Health Progress doi: 10.1094/PHP-2008-0318-01-RS.

\begin{abstract}
The genus Phytophthora includes species causing diseases such as late blight of potatoes, Phytophthora infestans, and sudden oak death and ramorum blight, P. ramorum. Because of the importance of diseases caused by Phytophthora, there is a need to have rapid access to the literature using their scientific names. The literature has been reviewed for all names in Phytophthora in order to provide the scientific name of each accepted species with authors and synonyms as well as the plant host range and worldwide geographic distribution. Within the genus Phytophthora, there are 87 accepted species and six infraspecific taxa. After compiling all available reports of Phytophthora, it was determined that 39 species and six infraspecific taxa, or about one-half of the accepted species, are not known to occur in the United States. The accurate scientific names of accepted species of Phytophthora are listed in two tables based on their presence or absence in the United States. Each species name is hyperlinked to databases that provide full synonymy and references documenting the host range and geographic distribution information. These data are continuously updated as new literature is published. Having rapid access to information about species of Phytophthora is critical for protecting the United States from the introduction of these potentially devastating pathogens.
\end{abstract}

The genus Phytophthora includes species causing diseases such as late blight of potatoes, Phytophthora infestans, and sudden oak death and ramorum blight, P. ramorum, which can inflict considerable damage on their hosts. The Irish potato famine in the 1840 s caused by P. infestans resulted in millions of deaths and triggered a significant ethnic migration (1). The full consequences of the introduction of Phytophthora ramorum to the western United States remain to be determined, but the financial impact is already staggering (2).

The recent advent of sudden oak death in Europe and western North America has sparked a resurgence of taxonomic interest in Phytophthora. Species of the genus Phytophthora were thoroughly and ably reviewed by Erwin \& Ribeiro in 1996 (1), who included 59 species with five varieties. Since that publication, however, 28 new species of Phytophthora or about one-third of the known taxa have been described along with a number of other taxonomic changes. As of December 2007, seven new species have been proposed but not yet formally described. The significant economic impact of diseases caused by Phytophthora spp., many of them recently described, has reinforced the need for an up-to-date synopsis of Phytophthora. 
Here we present a listing of the accepted species and infraspecific taxa currently included in Phytophthora based on the available taxonomic and plant pathology literature. For each species the published literature is summarized including the accepted scientific name with authors as well as a summary of the plant host range and worldwide geographic distribution. The authors of scientific names are abbreviated according to the recommendation of Brummitt \& Powell (3), now considered the standard for all organisms with nomenclature governed by the International Code of Botanical Nomenclature. Each name is hyperlinked to a more detailed account available at the website of the USDA Agricultural Research Service Systematic Mycology \&

Microbiology Laboratory (4). For each species data are provided that include the accepted scientific name with authors, synonyms, substrate affected, disease characteristics, plant host range, worldwide geographic distribution, and important recent literature, along with source literature and specimens that document this information. These databases are continuously updated as new literature is published, thus the hyperlinks allow the user to obtain recent information about each species of Phytophthora. In this paper we present a synopsis of these data as two tables including the scientific name with authors and a summary of host and geographic distribution. One table includes taxa that do not occur in the United States (Table 1) while the other lists taxa that occur in the United States (Table 2). In addition seven provisional names, four invalidly published names, and one potential name in Phythophthora are listed in Table 3. Many of the accepted species of Phytophthora have synonyms that are not listed in these tables but are included at the website. Some older reports were made using names now considered synonyms; thus, the ability to search using all synonymous names is critical in accurately determining the full host range and geographic distribution for a species. Access to updated information is especially important as surveys for Phytophthora provide new reports of species not previously known in the United States and throughout the world.

Currently, there are 87 accepted species and six infraspecific taxa in the genus Phytophthora. An additional seventy names in Phytophthora are treated as synonyms, 15 are referred to other genera, and at least 12 names have been proposed in the literature but have not been validly published. At present 47 taxa of Phytophthora or about half of the described taxa have not been reported in the United States. These include 40 species, one hybrid species, two subspecies, three varieties, and one forma specialis (Table 1). With increased surveys for Phytophthora, new records are being reported such as the two species, P. inundata and P. quercina, previously unknown in the United States reported to occur here in 2007 or the recent discovery of P. kernoviae in New Zealand by Ramsfield et al. (5).

Table 1. Phytophthora not reported in the United States.

\begin{tabular}{|l|l|l|}
\hline Scientific name & Hosts & $\begin{array}{l}\text { Geographic } \\
\text { distribution }\end{array}$ \\
\hline $\begin{array}{l}\text { Phytophthora alni Brasier \& } \\
\text { S.A. Kirk 2004 subsp. alni }\end{array}$ & Alnus spp. (Betulaceae) & Europe \\
\hline $\begin{array}{l}\text { Phytophthora alni subsp. } \\
\text { multiformis Brasier \& S.A. }\end{array}$ & Alnus spp. (Betulaceae) & $\begin{array}{l}\text { Europe (Germany, } \\
\text { the Netherlands, UK) }\end{array}$ \\
\hline Kirk 2004 & Phytophthora alni subsp. \\
\hline $\begin{array}{l}\text { uniformis Brasier \& S.A. Kirk } \\
2004\end{array}$ & Alnus spp. (Betulaceae) & Europe \\
\hline $\begin{array}{l}\text { Phytophthora alticola } \\
\text { Maseko, Coutinho \& M.J. }\end{array}$ & $\begin{array}{l}\text { Eucalyptus badjensis } \\
\text { (Myrtaceae) }\end{array}$ & South Africa \\
\hline
\end{tabular}




\begin{tabular}{|c|c|c|}
\hline Wingf. 2007 & & \\
\hline$\frac{\text { Phytophthora austrocedrae }}{\text { Greslebin \& Hansen } 2007}$ & $\begin{array}{l}\text { Austrocedrus chilensis } \\
\text { (Cupressaceae) }\end{array}$ & $\begin{array}{l}\text { South America } \\
\text { (Argentina) }\end{array}$ \\
\hline $\begin{array}{l}\text { Phytophthora boehmeriae } \\
\text { Sawada } 1927\end{array}$ & $\begin{array}{l}\text { Citrus spp. (Rutaceae) } \\
\text { and various other } \\
\text { families }\end{array}$ & $\begin{array}{l}\text { Africa (South Africa), } \\
\text { Asia, Australia and } \\
\text { Papua New Guinea, } \\
\text { Europe (Greece), } \\
\text { South America } \\
\text { (Argentina) }\end{array}$ \\
\hline$\frac{\text { Phytophthora botryosa }}{1969}$ & $\begin{array}{l}\text { Colocasia (Araceae), } \\
\text { Hevea (Euphorbiaceae); } \\
\text { when inoculated, } \\
\text { Theobroma cacao } \\
\text { (Malvaceae) }\end{array}$ & $\begin{array}{l}\text { Asia (China, Malaysia, } \\
\text { Thailand, Andaman } \\
\text { I slands, Vietnam) }\end{array}$ \\
\hline$\frac{\text { Phytophthora } \times \text { cactorum- }}{\text { nicotianae Man in 't Veld et }}$ & $\begin{array}{l}\text { Six genera in six } \\
\text { families, including } \\
\text { Cyclamen, Eriobotrya, } \\
\text { Lavandula, Lewisia, } \\
\text { Primula, Spathiphyllum }\end{array}$ & $\begin{array}{l}\text { Europe (the } \\
\text { Netherlands in } \\
\text { hydroponic } \\
\text { greenhouses), Asia } \\
\text { (Taiwan) }\end{array}$ \\
\hline $\begin{array}{l}\text { Phytophthora cajani K.M. } \\
\text { Amin, Baldev \& F.J. Williams } \\
1978\end{array}$ & $\begin{array}{l}\text { Cajanus cajani } \\
\text { (Fabaceae) }\end{array}$ & Asia (India) \\
\hline $\begin{array}{l}\text { Phytophthora canavaliae } \\
\text { Hara } 1932\end{array}$ & $\begin{array}{l}\text { Canavalia ensiformis } \\
\text { (Fabaceae) }\end{array}$ & Asia (Japan) \\
\hline $\begin{array}{l}\text { Phytophthora captiosa M.A. } \\
\text { Dick \& Dobbie } 2006\end{array}$ & $\begin{array}{l}\text { Eucalyptus botryoides, } \\
\text { E. saligna (Myrtaceae) }\end{array}$ & New Zealand \\
\hline $\begin{array}{l}\frac{\text { Phytophthora cinnamomi }}{\text { var. parvispora Kröber \& }} \\
\text { Marwitz } 1993\end{array}$ & $\begin{array}{l}\text { Beaucarnea sp. } \\
\text { (Agavaceae) }\end{array}$ & Europe (Germany) \\
\hline $\begin{array}{l}\text { Phytophthora cinnamomi } \\
\text { var. robiniae H.H. Ho } 2002\end{array}$ & $\begin{array}{l}\text { Robinia pseudoacacia } \\
\text { (Fabaceae) }\end{array}$ & Asia (China) \\
\hline $\begin{array}{l}\text { Phytophthora clandestina } \\
\text { P.A. Taylor, Pascoe \& F.C. } \\
\text { Greenh. } 1985\end{array}$ & $\begin{array}{l}\text { Medicago spp., Trifolium } \\
\text { subterraneum, } \\
\text { (Fabaceae) }\end{array}$ & Australia \\
\hline $\begin{array}{l}\text { Phytophthora colocasiae } \\
\text { Rabenh. } 1900\end{array}$ & $\begin{array}{l}\text { Colocasia esculenta } \\
\text { (taro) and other } \\
\text { Araceae; also six genera } \\
\text { in five other families }\end{array}$ & $\begin{array}{l}\text { Africa (Ethiopia, East } \\
\text { Africa, Fernando Po), } \\
\text { Asia, Caribbean } \\
\text { (Dominican } \\
\text { Republic), North } \\
\text { America (USA: CA, } \\
\text { NC, HI), Pacific } \\
\text { Islands, South } \\
\text { America (Brazil, } \\
\text { Argentina) }\end{array}$ \\
\hline $\begin{array}{l}\text { Phytophthora cyperi-bulbosi } \\
\text { Seethal. \& K. Ramakr. } 1953\end{array}$ & $\begin{array}{l}\text { Cyperus bulbosus } \\
\text { (Cyperaceae) }\end{array}$ & Asia (India) \\
\hline$\frac{\text { Phytophthora fallax M.A. }}{\text { Dick \& Dobbie } 2006}$ & $\begin{array}{l}\text { Eucalyptus botryoides, } \\
\text { E. saligna (Myrtaceae) }\end{array}$ & New Zealand \\
\hline $\begin{array}{l}\text { Phytophthora frigida } \\
\text { Maseko, Coutinho \& M.J. } \\
\text { Wingf. } 2007\end{array}$ & $\begin{array}{l}\text { Eucalyptus smithii } \\
\text { (Myrtaceae) }\end{array}$ & South Africa \\
\hline $\begin{array}{l}\text { Phytophthora humicola W.H. } \\
\text { Ko \& Ann } 1985\end{array}$ & $\begin{array}{l}\text { Natural host unknown, } \\
\text { isolated from Citrus }\end{array}$ & Asia (Taiwan) \\
\hline
\end{tabular}




\begin{tabular}{|c|c|c|}
\hline & $\begin{array}{l}\text { orchard (Rutaceae); } \\
\text { when inoculated, } \\
\text { Cucumis sativus } \\
\text { (Cucurbitaceae) and } \\
\text { Malus domestica } \\
\text { (Rosaceae) }\end{array}$ & \\
\hline$\frac{\text { Phytophthora idaei D.M. }}{\text { Kenn. } 1995}$ & Rubus idaeus (Rosaceae) & Europe (UK) \\
\hline $\begin{array}{l}\text { Phytophthora ipomoeae Flier } \\
\text { \& Grünwald } 2002\end{array}$ & $\begin{array}{l}\text { I pomoea } \\
\text { longipedunculata, I. } \\
\text { purpurea } \\
\text { (Convolvulaceae) }\end{array}$ & $\begin{array}{l}\text { North America } \\
\text { (Mexico) }\end{array}$ \\
\hline $\begin{array}{l}\text { Phytophthora iranica Ershad } \\
1971\end{array}$ & $\begin{array}{l}\text { Solanaceae; when } \\
\text { inoculated, Beta vulgaris } \\
\text { (Chenopodiaceae) }\end{array}$ & Asia (Iran) \\
\hline $\begin{array}{l}\text { Phytophthora italica } \\
\text { Cacciola, Magnano \& } \\
\text { Belisario } 1996\end{array}$ & $\begin{array}{l}\text { Myrtus communis } \\
\text { (Myrtaceae) }\end{array}$ & Europe (Italy) \\
\hline $\begin{array}{l}\text { Phytophthora japonica G.M. } \\
\text { Waterh. } 1974\end{array}$ & Oryza sativa (Poaceae) & Asia (Japan) \\
\hline $\begin{array}{l}\text { Phytophthora kernoviae } \\
\text { Brasier, Beales \& S.A. Kirk } \\
2005\end{array}$ & $\begin{array}{l}\text { Fagus sylvatica, Quercus } \\
\text { robur (Fagaceae), } \\
\text { Liriodendron tulipifera } \\
\text { (Magnoliaceae), } \\
\text { Rhododendron ponticum } \\
\text { (Ericaceae) }\end{array}$ & $\begin{array}{l}\text { Europe (UK); New } \\
\text { Zealand }\end{array}$ \\
\hline $\begin{array}{l}\text { Phytophthora leersiae } \\
\text { Sawada ex H.H. Ho \& H.S. } \\
\text { Chang } 1992\end{array}$ & $\begin{array}{l}\text { Leersia hexandra } \\
\text { (Poaceae) }\end{array}$ & Asia (China, Taiwan) \\
\hline $\begin{array}{l}\text { Phytophthora lepironiae } \\
\text { Sawada } 1919\end{array}$ & $\begin{array}{l}\text { Lepironia spp. } \\
\text { (Cyperaceae) }\end{array}$ & $\begin{array}{l}\text { Asia (China, Japan, } \\
\text { Korea, Taiwan) }\end{array}$ \\
\hline$\frac{\text { Phytophthora }}{\text { macrochlamydospora J.A.G. }}$ & Glycine max (Fabaceae) & Australia \\
\hline$\frac{\text { Phytophthora megakarya }}{\text { Brasier \& M.J. Griffin } 1979}$ & $\begin{array}{l}\text { I rvingia sp. } \\
\text { (Irvingiaceae) } \\
\text { Theobroma cacao } \\
\text { (Malvaceae), }\end{array}$ & West Africa \\
\hline $\begin{array}{l}\text { Phytophthora melonis } \\
\text { Katsura } 1976\end{array}$ & $\begin{array}{l}\text { Citrullus lanatus, } \\
\text { Cucumis sativus, } \\
\text { Trichosanthes dioica } \\
\text { (Cucurbitaceae), Pistacia } \\
\text { vera (Anacardiaceae) }\end{array}$ & $\begin{array}{l}\text { Asia (China, India, } \\
\text { Iran, Japan, Korea, } \\
\text { Taiwan) }\end{array}$ \\
\hline$\frac{\text { Phytophthora mexicana }}{\text { Hotson \& Hartge } 1923}$ & $\begin{array}{l}\text { Lycopersicon esculentum } \\
\text { (Solanaceae) }\end{array}$ & $\begin{array}{l}\text { North America } \\
\text { (Mexico), possibly } \\
\text { introduced from the } \\
\text { Netherlands (1) }\end{array}$ \\
\hline $\begin{array}{l}\text { Phytophthora mirabilis } \\
\text { Galindo \& H.R. Hohl } 1986\end{array}$ & $\begin{array}{l}\text { Mirabilis jalapa } \\
\text { ( Nyctaginaceae) }\end{array}$ & $\begin{array}{l}\text { North America } \\
\text { (Mexico) }\end{array}$ \\
\hline $\begin{array}{l}\text { Phytophthora } \\
\text { multivesiculata Ilieva, Man } \\
\text { in 't Veld, W. Veenb.-Rijks \& }\end{array}$ & $\begin{array}{l}\text { Cymbidium sp. } \\
\text { (Orchidaceae) }\end{array}$ & $\begin{array}{l}\text { Europe (the } \\
\text { Netherlands) }\end{array}$ \\
\hline
\end{tabular}




\begin{tabular}{|c|c|c|}
\hline R. Pieters 1998 & & \\
\hline $\begin{array}{l}\text { Phytophthora oryzo-bladis } \\
\text { J.S. Wang \& J.Y. Lu ex H.H. } \\
\text { Ho } 2001\end{array}$ & Oryza sativa (Poaceae) & Asia (China) \\
\hline $\begin{array}{l}\text { Phytophthora palmivora var. } \\
\text { heterocystica Babacauh } \\
1983\end{array}$ & $\begin{array}{l}\text { Theobroma cacao } \\
\text { (Malvaceae) }\end{array}$ & $\begin{array}{l}\text { Africa (I vory Coast); } \\
\text { may not be distinct } \\
\text { from P. palmivora }\end{array}$ \\
\hline$\frac{\text { Phytophthora pistaciae }}{\text { Mirab. } 2001}$ & $\begin{array}{l}\text { Pistacia vera } \\
\text { (Anacardiaceae) }\end{array}$ & Asia (Iran) \\
\hline $\begin{array}{l}\text { Phytophthora polonica } \\
\text { Belbahri, Moralejo \& Lefort } \\
2007\end{array}$ & $\begin{array}{l}\text { I solated from soil } \\
\text { associated with Alnus } \\
\text { glutinosa }\end{array}$ & Poland \\
\hline $\begin{array}{l}\text { Phytophthora polygoni } \\
\text { Sawada } 1922\end{array}$ & $\begin{array}{l}\text { Polygonum spp., Rumex } \\
\text { dentatus (Polygonaceae) }\end{array}$ & Asia (China, Taiwan) \\
\hline$\frac{\text { Phytophthora porri Foister }}{1931}$ & $\begin{array}{l}\text { Allium spp. (Liliaceae), } \\
\text { five other genera in five } \\
\text { families }\end{array}$ & $\begin{array}{l}\text { Africa (South Africa), } \\
\text { Asia (J apan), } \\
\text { Australia, Europe, } \\
\text { North America } \\
\text { (Canada). The report } \\
\text { from USA: WI is } \\
\text { actually Phytophthora } \\
\text { brassicae. }\end{array}$ \\
\hline$\frac{\text { Phytophthora primulae J.A. }}{\text { Tomlinson } 1952}$ & $\begin{array}{l}\text { Primula spp. } \\
\text { (Primulaceae) }\end{array}$ & $\begin{array}{l}\text { Europe (Denmark, } \\
\text { Germany, UK), New } \\
\text { Zealand }\end{array}$ \\
\hline$\frac{\text { Phytophthora psychrophila }}{\text { T. Jung \& E. M. Hansen } 2002}$ & $\begin{array}{l}\text { Ilex aquifolium } \\
\text { (Aquifoliaceae), Quercus } \\
\text { spp. (Fagaceae) when } \\
\text { inoculated }\end{array}$ & $\begin{array}{l}\text { Europe (France, } \\
\text { Germany, UK) }\end{array}$ \\
\hline$\frac{\text { Phytophthora quininea }}{\text { Crand. } 1947}$ & $\begin{array}{l}\text { Cinchona spp. } \\
\text { (Rubiaceae) }\end{array}$ & $\begin{array}{l}\text { Central America } \\
\text { (Guatemala), } \\
\text { Caribbean Islands } \\
\text { (Puerto Rico), South } \\
\text { America (Bolivia, } \\
\text { Peru) }\end{array}$ \\
\hline$\frac{\text { Phytophthora tentaculata }}{\text { Kröber \& Marwitz } 1993}$ & $\begin{array}{l}\text { Chrysanthemum spp., } \\
\text { Santolina (Asteraceae), } \\
\text { Verbena (Verbenaceae) }\end{array}$ & $\begin{array}{l}\text { Europe (Spain, } \\
\text { Germany) }\end{array}$ \\
\hline $\begin{array}{l}\text { Phytophthora uliginosa T. } \\
\text { J ung \& E. M. Hansen } 2002\end{array}$ & $\begin{array}{l}\text { Quercus robur, Quercus } \\
\text { petraea (Fagaceae) }\end{array}$ & $\begin{array}{l}\text { Europe (Poland, } \\
\text { Germany) }\end{array}$ \\
\hline $\begin{array}{l}\text { Phytophthora verrucosa } \\
\text { Alcock \& Foister } 1940\end{array}$ & $\begin{array}{l}\text { Four families including } \\
\text { Lycopersicon esculentum } \\
\text { (Solanaceae) }\end{array}$ & Europe (UK) \\
\hline$\frac{\text { Phytophthora vignae Purss }}{1957}$ & $\begin{array}{l}\text { Cajanus cajani, Vigna } \\
\text { spp. } \\
\text { (Fabaceae); when } \\
\text { inoculated, Malus } \\
\text { domestica (Rosaceae) } \\
\text { and Solanum tuberosum } \\
\text { (Solanaceae) }\end{array}$ & $\begin{array}{l}\text { Asia (China, Hong } \\
\text { Kong, India, Japan, } \\
\text { Sri Lanka, Taiwan), } \\
\text { Australia }\end{array}$ \\
\hline $\begin{array}{l}\text { Phytophthora vignae f. sp. } \\
\text { adzukicola S. Tsuchiya, } \\
\text { Yanagawa \& Ogoshi } 1986\end{array}$ & $\begin{array}{l}\text { Phaseolus radiatus var. } \\
\text { aurea, Vigna angularis } \\
\text { (Fabaceae) }\end{array}$ & Asi \\
\hline
\end{tabular}


The synopsis of Phytophthora not known to occur in the United States provides a framework to guide more extensive investigations of the biology and geographic distribution of these important organisms especially for those charged with preventing their entry into the United States. Which of these species pose a threat to the United States as invasive species? One might assume that species most threatening are those that occur in temperate regions on perennial hosts such as the recently described taxa on trees in the Betulaceae, i.e., $P$. alni and its subspecies, those having a broad host range such as P. kernoviae on Ericaceae, Fagaceae, and Magnoliaceae, or those on perennial crops such as P. idaei on Rubus idaeus. Species that are known to occur close to US borders such as P. mexicana on Lycopersicon esculenta also pose a significant threat.

The species of Phytophthora already known to occur in the United States are listed in Table 2. Many of these species such as P. cinnamomi and P. cryptogea are cosmopolitan reported on a wide range of host plants. Others occur on only a restricted number of hosts such as P. ilicis on Ilex spp. or have been reported once in the US such as P. hibernalis on Citrus reported only from California or P. heveae reported on a wide range of hosts, primarily tropical ones, but also known from North Carolina and Tennessee in the US. A few species of Phytophthora are known only from Hawaii, specifically P. katsurae reported throughout the tropics on diverse woody plants and $\mathrm{P}$. meadii reported in Asia and Australia on various plant families. Known previously only from Europe, Phytophthora quercina was recently isolated from soil under declining oaks in Missouri (6) as was P. inundata from soil in California (7).

Table 2. Phytophthora reported in the United States.

\begin{tabular}{|c|c|c|}
\hline Scientific name & Hosts & Geographic distribution \\
\hline $\begin{array}{l}\text { Phytophthora } \\
\text { brassicae DeCock } \\
\text { \& Man in't Veld } \\
2002\end{array}$ & $\begin{array}{l}\text { Brassica oleracea and B. } \\
\text { sinenses (Brassicaceae) }\end{array}$ & $\begin{array}{l}\text { Europe (Germany, UK); } \\
\text { North America (USA: WI) }\end{array}$ \\
\hline $\begin{array}{l}\text { Phytophthora } \\
\text { cactorum (Lebert } \\
\text { \& Cohn) J. Schröt. } \\
1886\end{array}$ & 154 genera in 54 families & Cosmopolitan \\
\hline $\begin{array}{l}\text { Phytophthora } \\
\text { cambivora (Petri) } \\
\text { Buisman } 1927\end{array}$ & 30 genera in 19 families & Cosmopolitan \\
\hline$\frac{\text { Phytophthora }}{\frac{\text { capsici Leonian }}{1922}}$ & $\begin{array}{l}\text { P. capsici sensu lato infects } \\
51 \text { genera in } 28 \text { families, } \\
\text { including Capsicum annuum, } \\
\text { Lycopersicon esculentum } \\
\text { and other Solanaceae. } \\
\text { Aragaki \& Uchida (9) refer } \\
\text { isolates from non-Capsicum } \\
\text { hosts to P. tropicalis }\end{array}$ & $\begin{array}{l}\text { Cosmopolitan. Tropical } \\
\text { species may now be } \\
\text { considered P. tropicalis }\end{array}$ \\
\hline $\begin{array}{l}\text { Phytophthora } \\
\text { cinnamomi Rands } \\
\begin{array}{l}1922 \text { var. } \\
\text { cinnamomi }\end{array}\end{array}$ & $\begin{array}{l}266 \text { genera in } 90 \text { families, } \\
\text { commonly hardwood trees }\end{array}$ & Cosmopolitan \\
\hline $\begin{array}{l}\text { Phytophthora } \\
\frac{\text { citricola Sawada }}{1927}\end{array}$ & 75 genera in 38 families & Cosmopolitan \\
\hline
\end{tabular}




\begin{tabular}{|c|c|c|}
\hline $\begin{array}{l}\text { Phytophthora } \\
\text { citrophthora (R.E. } \\
\text { Sm. \& E.H. Sm.) } \\
\text { Leonian } 1906\end{array}$ & 88 genera in 51 families & Cosmopolitan \\
\hline $\begin{array}{l}\text { Phytophthora } \\
\text { cryptogea } \\
\text { Pethybr. \& Laff. } \\
1919\end{array}$ & 141 genera in 49 families & Cosmopolitan \\
\hline$\frac{\text { Phytophthora }}{\frac{\text { cyperi (Ideta) I to }}{1935}}$ & $\begin{array}{l}\text { Cyperus spp. (Cyperaceae), } \\
\text { Digitaria ciliaris (Poaceae) }\end{array}$ & $\begin{array}{l}\text { Africa (Sudan), Asia, Europe } \\
\text { (UK), North America (USA: } \\
\text { SC, TX) }\end{array}$ \\
\hline $\begin{array}{l}\frac{\text { Phytophthora }}{\text { drechsleri Tucker }} \\
1931\end{array}$ & 113 genera in 40 families & Cosmopolitan \\
\hline$\frac{\text { Phytophthora }}{\text { erythroseptica }}$ & $\begin{array}{l}\text { Principal hosts Solanaceae, } \\
15 \text { genera in } 10 \text { other } \\
\text { families }\end{array}$ & Cosmopolitan \\
\hline $\begin{array}{l}\text { Phytophthora } \\
\text { europaea E.M. } \\
\text { Hansen \& T. Jung } \\
2002\end{array}$ & Quercus spp. (Fagaceae) & $\begin{array}{l}\text { Europe (France, Germany), } \\
\text { North America (USA) }\end{array}$ \\
\hline $\begin{array}{l}\text { Phytophthora } \\
\frac{\text { foliorum Donahoo }}{\& \text { Lamour } 2006}\end{array}$ & $\begin{array}{l}\text { Rhododendron sp. } \\
\text { (Ericaceae) }\end{array}$ & $\begin{array}{l}\text { North America (USA: CA, } \\
\text { TN) }\end{array}$ \\
\hline $\begin{array}{l}\text { Phytophthora } \\
\text { fragariae Hickman } \\
\underline{1940 \text { var. }} \\
\underline{\text { fragariae }}\end{array}$ & $\begin{array}{l}\text { Fragaria ×ananassa and } \\
\text { Rubus ursinus var. } \\
\text { longanobaccus (Rosaceae); } \\
\text { when inoculated, other } \\
\text { Rosaceae, Chenopodiaceae, } \\
\text { and Solanaceae on (1) }\end{array}$ & $\begin{array}{l}\text { Asia, Australia, New } \\
\text { Zealand, Europe, North } \\
\text { America (Canada, USA) }\end{array}$ \\
\hline $\begin{array}{l}\text { Phytophthora } \\
\text { gonapodyides } \\
\text { (H.E. Petersen) } \\
\text { Buisman } 1927\end{array}$ & $\begin{array}{l}13 \text { genera in } 11 \text { families, } \\
\text { including Malus spp. } \\
\text { (Rosaceae), also on } \\
\text { Pinaceae seedlings including } \\
\text { Abies, Pseudotsuga } \\
\text { menziesii, and Tsuga } \\
\text { mertensiana }\end{array}$ & $\begin{array}{l}\text { Australia, New Zealand, } \\
\text { Europe (Denmark), North } \\
\text { America (USA), South } \\
\text { America (Chile) }\end{array}$ \\
\hline $\begin{array}{l}\text { Phytophthora } \\
\text { hedraiandra De } \\
\text { Cock \& Man in 't } \\
\text { Veld } 2004\end{array}$ & $\begin{array}{l}\text { Rhododendron (Ericaceae), } \\
\text { Viburnum (Adoxaceae), }\end{array}$ & $\begin{array}{l}\text { Europe (the Netherlands, } \\
\text { Slovakia), North America } \\
\text { (USA: MN) }\end{array}$ \\
\hline $\begin{array}{l}\frac{\text { Phytophthora }}{\text { heveae A. }} \\
\text { Thomps. } 1929\end{array}$ & $\begin{array}{l}12 \text { genera in } 11 \text { families } \\
\text { including Cocos nucifera } \\
\text { (Arecaceae), Hevea } \\
\text { (Euphorbiaceae) and } \\
\text { Rhododendron (Ericaceae) }\end{array}$ & $\begin{array}{l}\text { Asia, Australasia, North } \\
\text { America (USA: NC, TN), } \\
\text { South America (Brazil) }\end{array}$ \\
\hline $\begin{array}{l}\frac{\text { Phytophthora }}{\text { hibernalis Carne }} \\
1925\end{array}$ & $\begin{array}{l}\text { Citrus spp. (Rutaceae), also } \\
11 \text { genera in } 9 \text { other families }\end{array}$ & $\begin{array}{l}\text { Africa (South Africa); Asia } \\
\text { (Israel); Australasia; } \\
\text { Europe; North America } \\
\text { (USA: CA, OR); Central } \\
\text { America, Caribbean Islands; } \\
\text { South America (Argentina, } \\
\text { Brazil, Venezuela) }\end{array}$ \\
\hline Phytophthora ilicis & Ilex spp. (Aquifoliaceae) & Europe (UK), North America \\
\hline
\end{tabular}




\begin{tabular}{|c|c|c|}
\hline $\begin{array}{l}\text { Buddenhagen \& } \\
\text { R.A. Young } 1957\end{array}$ & & (Canada, USA) \\
\hline $\begin{array}{l}\text { Phytophthora } \\
\text { infestans (Mont.) } \\
\text { de Bary } 1876\end{array}$ & $\begin{array}{l}\text { Principal hosts Solanaceae, } \\
\text { also on } 15 \text { genera in ten } \\
\text { other families }\end{array}$ & Cosmopolitan \\
\hline $\begin{array}{l}\text { Phytophthora } \\
\text { inflata Caroselli \& } \\
\text { Tucker } 1949\end{array}$ & $\begin{array}{l}\text { Ulmus spp. (Ulmaceae), also } \\
\text { on Rhododendron, } \\
\text { Sambucus and Syringa }\end{array}$ & $\begin{array}{l}\text { Europe (England), North } \\
\text { America (Canada, USA) }\end{array}$ \\
\hline $\begin{array}{l}\text { Phytophthora } \\
\text { insolita Ann \& } \\
\text { W.H. Ko } 1981\end{array}$ & $\begin{array}{l}\text { Rhododendron (Ericaceae); } \\
\text { when inoculated, on fruits of } \\
\text { Cucumis sativus } \\
\text { (Cucurbitaceae) and Malus } \\
\text { domestica (Rosaceae) }\end{array}$ & $\begin{array}{l}\text { Asia (Taiwan), North } \\
\text { America (USA: OH) }\end{array}$ \\
\hline $\begin{array}{l}\text { Phytophthora } \\
\text { inundata Brasier } \\
\text { et al. } 2003\end{array}$ & $\begin{array}{l}\text { Various families, including } \\
\text { Aesculus hippocastanum } \\
\text { (Sapindaceae), Olea } \\
\text { (Oleaceae), Salix } \\
\text { (Salicaceae), and Vitis } \\
\text { (Vitaceae) }\end{array}$ & $\begin{array}{l}\text { Europe (Denmark, France, } \\
\text { Spain, UK), South America, } \\
\text { recently reported in USA } \\
\text { (CA) }\end{array}$ \\
\hline $\begin{array}{l}\text { Phytophthora } \\
\text { katsurae W.H. Ko } \\
\text { \& H.S. Chang } \\
1979\end{array}$ & $\begin{array}{l}\text { Castanea crenata } \\
\text { (Fagaceae), Cocos nucifera } \\
\text { (Arecaceae), Theobroma } \\
\text { cacao (Malvaceae) }\end{array}$ & $\begin{array}{l}\text { Africa (Ivory Coast), Asia } \\
\text { (Japan, Korea, Taiwan), } \\
\text { Caribbean (J amaica), Pacific } \\
\text { Islands (USA: HI), Papua } \\
\text { New Guinea }\end{array}$ \\
\hline$\frac{\text { Phytophthora }}{\text { Iateralis Tucker \& }}$ & $\begin{array}{l}\text { Chamaecyparis lawsoniana } \\
\text { (Cupressaceae), rarely, } \\
\text { Taxus brevifolia (Taxaceae); } \\
\text { when inoculated, hosts in } \\
\text { other families }\end{array}$ & $\begin{array}{l}\text { Europe (France), North } \\
\text { America (Canada, USA). } \\
\text { Reports from New Zealand } \\
\text { are doubtful. }\end{array}$ \\
\hline $\begin{array}{l}\text { Phytophthora } \\
\frac{\text { meadii McRae }}{1918}\end{array}$ & $\begin{array}{l}\text { Various families including } \\
\text { Hevea spp. (Euphorbiaceae) }\end{array}$ & $\begin{array}{l}\text { Asia, Australia, Pacific } \\
\text { Islands (USA: HI) }\end{array}$ \\
\hline $\begin{array}{l}\text { Phytophthora } \\
\text { medicaginis E.M. } \\
\text { Hansen \& D.P. } \\
\text { Maxwell } 1991\end{array}$ & $\begin{array}{l}\text { Cicer arietinum, Medicago } \\
\text { sativa, Onobrychis viciifolia } \\
\text { (Fabaceae), Prunus mahaleb } \\
\text { (Rosaceae) }\end{array}$ & Cosmopolitan \\
\hline$\frac{\text { Phytophthora }}{\text { megasperma }}$ & $\begin{array}{l}\text { Fabaceae and many other } \\
\text { families; isolates previously } \\
\text { referred to as P. } \\
\text { megasperma are now } \\
\text { considered to be three } \\
\text { distinct species }(10,11)\end{array}$ & Cosmopolitan \\
\hline $\begin{array}{l}\text { Phytophthora } \\
\text { nemorosa E.M. } \\
\text { Hansen \& Reeser } \\
2003\end{array}$ & $\begin{array}{l}\text { Various families, including } \\
\text { Lithocarpus densiflorus, } \\
\text { Quercus agrifolia (Fagaceae) }\end{array}$ & $\begin{array}{l}\text { North America (USA: CA, } \\
\text { OR) }\end{array}$ \\
\hline $\begin{array}{l}\text { Phytophthora } \\
\text { nicotianae Breda } \\
\text { de Haan } 1896\end{array}$ & 255 genera in 90 families & Cosmopolitan \\
\hline $\begin{array}{l}\text { Phytophthora } \\
\text { palmivora (E.J. } \\
\text { Butler) E.J. Butler } \\
1919 \text { var. } \\
\text { palmivora }\end{array}$ & 160 genera in 60 families & Cosmopolitan \\
\hline
\end{tabular}




\begin{tabular}{|c|c|c|}
\hline $\begin{array}{l}\text { Phytophthora } \\
\text { phaseoli Thaxt. } \\
1889\end{array}$ & $\begin{array}{l}\text { Phaseolus lunatus, } \mathrm{P} \text {. } \\
\text { vulgaris (Fabaceae), other } \\
\text { families after inoculation ( } 1 \text { ) }\end{array}$ & $\begin{array}{l}\text { Africa (Congo, Zaire), Asia } \\
\text { (Philippines, Sri Lanka, } \\
\text { USSR), Europe (Italy, } \\
\text { Romania), North America } \\
\text { (Mexico, USA), Central } \\
\text { America and Caribbean } \\
\text { Islands, South America } \\
\text { (Brazil) }\end{array}$ \\
\hline $\begin{array}{l}\frac{\text { Phytophthora }}{\text { pseudosyringae }} \mathrm{T} \\
\text { Jung \& Delatour } \\
2003\end{array}$ & $\begin{array}{l}\text { Alnus glutinosa, Fagus } \\
\text { sylvatica, Quercus spp. , and } \\
\text { other hardwood species }\end{array}$ & $\begin{array}{l}\text { Germany, Spain, North } \\
\text { America (USA: CA) }\end{array}$ \\
\hline $\begin{array}{l}\frac{\text { Phytophthora }}{\text { pseudotsugae }} \\
\text { Hamm \& E.M. } \\
\text { Hansen } 1983\end{array}$ & $\begin{array}{l}\text { Pseudotsuga menziesii } \\
\text { (Pinaceae) }\end{array}$ & $\begin{array}{l}\text { North America (USA: OR, } \\
\text { WA) }\end{array}$ \\
\hline$\frac{\text { Phytophthora }}{\text { quercina T. Jung }}$ & Quercus spp. (Fagaceae) & $\begin{array}{l}\text { Asia (Turkey), Europe } \\
\text { (Germany, Hungary, Italy); } \\
\text { recently isolated from soil } \\
\text { under oak in North America } \\
\text { (USA: MO) (4) }\end{array}$ \\
\hline $\begin{array}{l}\text { Phytophthora } \\
\text { ramorum Werres, } \\
\text { De Cock \& Man in } \\
\text { 't Veld } 2001\end{array}$ & $\begin{array}{l}26 \text { genera in } 17 \text { families, } \\
\text { including Camellia, } \\
\text { Rhododendron (Ericaceae) } \\
\text { and Quercus (Fagaceae) }\end{array}$ & $\begin{array}{l}\text { Europe, North America } \\
\text { (Canada, USA: CA, OR, WA) }\end{array}$ \\
\hline$\frac{\text { Phytophthora }}{\text { richardiae }}$ & $\begin{array}{l}\text { Asparagus (Asparagaceae), } \\
\text { Daucus carota (Apiaceae), } \\
\text { Lycopersicon esculentum } \\
\text { (Solanaceae), Robinia } \\
\text { (Fabaceae), and } \\
\text { Zantedeschia (Araceae) }\end{array}$ & $\begin{array}{l}\text { Asia, Australasia, Australia, } \\
\text { Europe, North America } \\
\text { (USA) }\end{array}$ \\
\hline $\begin{array}{l}\text { Phytophthora rubi } \\
\text { (W.F. Wilcox \& } \\
\text { J.M. Duncan) Man } \\
\text { in 't Veld } 2007\end{array}$ & $\begin{array}{l}\text { Rubus idaeus var. idaeus } \\
\text { (Rosaceae) }\end{array}$ & Cosmopolitan \\
\hline $\begin{array}{l}\text { Phytophthora } \\
\text { Siskiyouensis } \\
\text { Reeser \& E.M. } \\
\text { Hansen } 2007 \text { (12) }\end{array}$ & $\begin{array}{l}\text { Isolated from soil and water } \\
\text { using Lithocarpus densiflorus } \\
\text { (tanoak) (Fagaceae) and } \\
\text { Rhododendron spp. } \\
\text { (Ericaceae) as leaf baits; } \\
\text { also reported from Alnus. }\end{array}$ & $\begin{array}{l}\text { North America (USA: CA, } \\
\text { OR); Australia }\end{array}$ \\
\hline $\begin{array}{l}\frac{\text { Phytophthora }}{\text { sojae Kaufm. \& }} \\
\text { Gerd. } 1958\end{array}$ & $\begin{array}{l}\text { Glycine max, Lupinus } \\
\text { (Fabaceae); also reported } \\
\text { from six other genera in five } \\
\text { families }\end{array}$ & $\begin{array}{l}\text { Australia, New Zealand, Asia } \\
\text { (China, Korea), North } \\
\text { America (Canada, USA), } \\
\text { South America (Brazil, } \\
\text { Chile) }\end{array}$ \\
\hline $\begin{array}{l}\text { Phytophthora } \\
\text { Syringae (Berk.) } \\
\text { Kleb. } 1909\end{array}$ & $\begin{array}{l}29 \text { genera in } 14 \text { families, } \\
\text { including Syringa vulgaris } \\
\text { (Oleaceae) }\end{array}$ & $\begin{array}{l}\text { Africa (Morocco, South } \\
\text { Africa); Asia (Korea) } \\
\text { Australasia; Europe; North } \\
\text { America (Canada, USA); } \\
\text { South America (Argentina, } \\
\text { Brazil, Peru) }\end{array}$ \\
\hline $\begin{array}{l}\text { Phytophthora } \\
\text { trifoliiE. M. Hansen } \\
\text { \& D.P. Maxwell } \\
1991\end{array}$ & Trifolium (Fabaceae) & North America (USA: MS) \\
\hline
\end{tabular}




\begin{tabular}{|c|c|c|}
\hline $\begin{array}{l}\text { Phytophthora } \\
\text { tropicalis Aragaki } \\
\text { \& J .Y. Uchida } \\
2001\end{array}$ & 14 genera in 12 families & $\begin{array}{l}\text { Pacific Islands (USA: HI), } \\
\text { North America (USA: VA, } \\
\text { Europe (the Netherlands, } \\
\text { Italy), before } 2001 \text { reported } \\
\text { as Phytophthora capsici, } \\
\text { thus geographic distribution } \\
\text { unclear }\end{array}$ \\
\hline
\end{tabular}

Table 3. Provisional and invalid Phytophthora names.

\begin{tabular}{|c|c|c|c|}
\hline Name & Status & $\begin{array}{l}\text { Host and/ or } \\
\text { substrate }\end{array}$ & Location \\
\hline $\begin{array}{l}\frac{\text { Phytophthora }}{\text { andina Kroon et al. }} \\
2004(11)\end{array}$ & $\begin{array}{l}\text { Provisional } \\
\text { name }\end{array}$ & $\begin{array}{l}\text { Solanum spp. } \\
\text { (Solanaceae) }\end{array}$ & South America \\
\hline $\begin{array}{l}\text { "Phytophthora } \\
\text { asparagi" Chimento } \\
\text { et al. } 2005 \text { (13) }\end{array}$ & $\begin{array}{l}\text { Provisional } \\
\text { name }\end{array}$ & $\begin{array}{l}\text { Agave sp. } \\
\text { (Agavaceae), } \\
\text { Asparagus sp. } \\
\text { (Asparagaceae), }\end{array}$ & Cosmopolitan \\
\hline $\begin{array}{l}\text { "Phytophthora } \\
\text { bisheria" Blair et al. } \\
2006 \text { (14) }\end{array}$ & $\begin{array}{l}\text { Provisional } \\
\text { name }\end{array}$ & $\begin{array}{l}\text { Fragaria ×ananasa, } \\
\text { Rosa sp., Rubus } \\
\text { idaeus (Rosaceae) }\end{array}$ & $\begin{array}{l}\text { Australia, Europe } \\
\text { (the } \\
\text { Netherlands), } \\
\text { North America } \\
\text { (USA: NC) }\end{array}$ \\
\hline $\begin{array}{l}\text { Phytophthora } \\
\text { eriugena Clancy \& } \\
\text { Kavanagh, nom. } \\
\text { inval. } 1979\end{array}$ & $\begin{array}{l}\text { I nvalidly } \\
\text { published, no } \\
\text { Latin diagnosis }\end{array}$ & $\begin{array}{l}\text { Chamaecyparis } \\
\text { lawsoniana } \\
\text { (Cupressaceae) }\end{array}$ & $\begin{array}{l}\text { Europe (I reland, } \\
\text { known only from } \\
\text { the type) }\end{array}$ \\
\hline $\begin{array}{l}\text { Phytophthora } \\
\text { erythroseptica var. } \\
\text { pisi" Bywater \& } \\
\text { Hickman, nom. } \\
\text { inval. } 1959\end{array}$ & $\begin{array}{l}\text { Invalidly } \\
\text { published, no } \\
\text { type specimen } \\
\text { designated }\end{array}$ & $\begin{array}{l}\text { Pisum sativum } \\
\text { (Fabaceae) }\end{array}$ & $\begin{array}{l}\text { Europe } \\
\text { (England) }\end{array}$ \\
\hline $\begin{array}{l}\text { "Phytophthora } \\
\text { glovera" Abad, } \\
\text { pers. comm. }\end{array}$ & $\begin{array}{l}\text { Provisional } \\
\text { name }\end{array}$ & $\begin{array}{l}\text { Nicotiana tabacum } \\
\text { (Solanaceae) }\end{array}$ & $\begin{array}{l}\text { South America } \\
\text { (Brazil) }\end{array}$ \\
\hline $\begin{array}{l}\text { Phytophthora } \\
\text { irritabilis Mantri \& } \\
\text { K.B. Deshp., nom. } \\
\text { inval. } 1968\end{array}$ & $\begin{array}{l}\text { Invalidly } \\
\text { published, no } \\
\text { type specimen } \\
\text { designated }\end{array}$ & $\begin{array}{l}\text { Phaseolus vulgaris } \\
\text { (Fabaceae) }\end{array}$ & Asia (India) \\
\hline $\begin{array}{l}\text { "Phytophthora } \\
\text { kelmania" Abad et } \\
\text { al. } 2006 \text { (15) }\end{array}$ & $\begin{array}{l}\text { Provisional } \\
\text { name }\end{array}$ & $\begin{array}{l}\text { Abies spp., Picea } \\
\text { spp. (Pinaceae), } \\
\text { Gerbera sp. } \\
\text { (Asteraceae), Coleus } \\
\text { sp. (Lamiaceae) }\end{array}$ & $\begin{array}{l}\text { Europe (Spain), } \\
\text { North America } \\
\text { (USA: NC) }\end{array}$ \\
\hline $\begin{array}{l}\text { Peronophythora } \\
\text { litchi Blair et al. } \\
2006(14)\end{array}$ & $\begin{array}{l}\text { May belong in } \\
\text { Phytophthora }\end{array}$ & $\begin{array}{l}\text { Litchi sp. } \\
\text { (Sapindaceae) }\end{array}$ & $\begin{array}{l}\text { Asia, Europe } \\
\text { (the } \\
\text { Netherlands) }\end{array}$ \\
\hline $\begin{array}{l}\text { "Phytophthora } \\
\text { niederhauseri" Abad } \\
\text { et al. } 2006 \text { (15) }\end{array}$ & $\begin{array}{l}\text { Provisional } \\
\text { name }\end{array}$ & $\begin{array}{l}\text { Various genera in } \\
\text { multiple families }\end{array}$ & $\begin{array}{l}\text { Australia, Europe } \\
\text { (Spain), North } \\
\text { America (USA: } \\
\text { NC) }\end{array}$ \\
\hline $\begin{array}{l}\text { "Phytophthora } \\
\text { persoonii" Abad et }\end{array}$ & $\begin{array}{l}\text { Provisional } \\
\text { name }\end{array}$ & $\begin{array}{l}\text { Nicotiana tabacum } \\
\text { (Solanaceae) }\end{array}$ & $\begin{array}{l}\text { North America } \\
\text { (USA: NC) }\end{array}$ \\
\hline
\end{tabular}




\begin{tabular}{|l|l|l|l|}
\hline al. 2006 (15) & & \\
\hline $\begin{array}{l}\text { "Phytophthora } \\
\text { rubra" Mantri \& K.B. } \\
\begin{array}{l}\text { Deshp., nom. inval. } \\
1968\end{array}\end{array}$ & $\begin{array}{l}\text { Invalidly } \\
\text { published, no } \\
\text { type specimen } \\
\text { designated }\end{array}$ & $\begin{array}{l}\text { Phaseolus vulgaris } \\
\text { (Fabaceae) }\end{array}$ & Asia (India) \\
\hline
\end{tabular}

Data synthesized in this document and presented through the hyperlinks are complete as of 20 December 2007 and will be updated on-line as new data are published. The authors welcome input and suggestions for missing or erroneous data and will make changes to the databases as necessary. With the intense scrutiny that the genus Phytophthora is receiving, changes are inevitable in the distribution of species, discovery of new taxa, and revision of species concepts as currently conceived. Of special note is the progress made in refining the species concepts within Phytophthora especially with the application of molecular systematic tools. Species concepts have changed to reflect their phylogeny and data for some species based on the literature is not accurate. For example, in molecular analyses Phytophthora undulata (H.E. Petersen) M.W. Dick 1989 clusters within the genus Pythium (8), and this species should be rereferred to by original name Pythium undulatum H.E. Petersen 1910. Although for some species of Phytophthora accurate identifications can be made based on morphology, increasingly identification requires sequencing of specific gene regions and "blasting" against databases of known sequences such as that available at the Phytophthora database at Pennsylvania State University.

This publication is intended to focus attention on those species of Phytophthora not yet present in the United States in the effort to safeguard US agriculture and protect native ecosystems. Accurate information about scientific names, geographic distribution, and plant host range provides the basis for tracking the movement and effective protection from introduction of invasive plant pathogens.

Literature Cited

1. Erwin, D. C., and Ribeiro, O. K. 1996. Phytophthora Diseases Worldwide. American Phytopathological Society, St. Paul, MN.

2. Reinherd, C. 2006. Impact of Phytophthora ramorum upon regulatory activities in Western states. Phytopathology 96:S146.

3. Brummitt, R. K., and Powell, C. E. 1992. Authors of Plant Names. Kew Royal Botanical Gardens, Cumbria, UK.

2. Farr, D. F., Rossman, A. Y., Palm, M. E., and McCray, E. B. 2008. Online. Fungal Databases, Systematic Botany \& Mycology Laboratory, ARS, USDA.

5. Ramsfield, T. D., Dick, M. A., Beever, R. E., and Horner, I. J . 2007. Phytophthora kernoviae - of southern hemisphere origin? Online. Fourth IUFRO Working Party Meeting on Phytophthoras in Forests and Natural Ecosystema, 26-31 August 2007, Monterey, Calif. Coll. of Nat. Resourc., Univ. of Calif., Berkeley.

6. Schwingle, B. W., J uzwik, J., and Moltzan, B. 2007. Phytophthora species in soils associated with declining and nondeclining oaks in Missouri forests. Plant Dis. 91:633.

7. Ho, H. H., Hong, C. X., and Erwin, D. C. 2006. Phytophthora inundata isolated from diseased alfalfa roots in southern California. Mycotaxon 97:349-358.

8. Villa, N. O., Kageyama, K., Asano, T., and Suga, H. 2006. Phylogenetic relationships of Pythium and Phytophthora species based on ITS rDNA, cytochrome oxidase II and beta-tubulin gene sequences. Mycologia 98:410-422.

9. Aragaki, M., and Uchida, J. Y. 2001. Morphological distinctions between Phytophthora capsici and P. tropicalis sp. nov. Mycologia 93:137-145.

10. Cooke, D. E. L., Drenth, A., Duncan, J . M., Wagels, G., and Brasier, C. M. 2000. A molecular phylogeny of Phytophthora and related Oomycetes. Fungal Genet. Biol. 30:17-32. 
11. Kroon, L. P. N. M., Bakker, F. T., van den Bosch, G. B. M., Bonants, P. J . M., and Flier, W. G. 2004. Phylogenetic analysis of Phytophthora species based on mitochondrial and nuclear DNS sequences. Fungal Genet. Biol. 41:766-782.

12. Reeser, P. W., Hansen, E. M., and Sutton, W. L. 2007. Phytophthora siskiyouensis, a new species from soil, water, myrtlewood (Umbellularia californica) and tanoak (Lithocarpus densiflorus) in southwestern Oregon. Mycologia 99:639-643.

13. Chimento, A., Scibetta, S., Schena, L., Cacciola, S. O., Green, K. R., and Cooke, D. E. L. 2005. The detection of Phytophthora in asparagus. J. Plant Path. 87:S291.

14. Blair, J. E., Kang, S., Geiser, D. M., and Coffey, M. 2006. A genus-wide phylogeny for Phytophthora utilizing whole genome sequence data. Phytopathology 96:S12.

15. Abad, Z. A., Abad, J . A., and Creswell, T. 2006. Species of Phytophthora and Pythium identified in a long term collection from North Carolina. Phytopathology 96:S1. 